\title{
ON CONVERGENCE FACTORS IN MULTIPLE SERIES*
}

\author{
BY \\ CHARLES N. MOORE
}

1. Introduction. Convergence factors may be defined as a set of functions of one or more parameters which, when introduced as factors of the terms of a series, cause a divergent series to converge or a series which is already convergent to converge more rapidly throughout a given range of values of the parameters. In the case of the convergence factors generally used in practice it is further true that each factor approaches unity as the parameters approach certain limit-values. Furthermore the function defined by the series for the given range of values of the parameters approaches a definite limit as the parameters approach the limitvalues, this limit being the value of the series for convergent series and a value we find it useful to ascribe to the series in the case of a divergent series. In the following discussion we shall find it convenient to distinguish between the cases where convergence factors merely have the property of reducing a series to convergence for a certain range of values of their parameters and the case where they have the additional property of obtaining a value for the series by the process of allowing the parameters to approach certain limit-values. We will refer to convergence factors that are only known to have the first property as being of type I, and convergence factors that have both properties as being of type II.

Several sets of sufficient conditions for convergence factors of type II in single series that are summable by Cesàro's method have been obtained by various writers. $\dagger$ The most general of these sets is that due to Bromwich. Hurwitz has obtained a set of necessary and sufficient conditions for this case, $\ddagger$ which are quite similar in form to Bromwich's conditions,

*Presented to the Society, December 28, 1923 and September 11, 1925; received by the editors before October, 1925.

†Cf. for example L. Fejér, Mathematische Annalen, vol. 58 (1904), p. 51; G. H. Hardy, Proceedings of the London Mathematical Society, (2), vol. 4 (1906), p. 247; Mathematische Annalen, vol. 64 (1907), p. 77; C. N. Moore, these Transactions, vol. 8 (1907), p. 299; T. J. Bromwich, Mathematische Annalen, vol. 65 (1908), p. 350; S. Chapman, Proceedings of the London Mathematical Society, (2), vol. 9 (1911), p. 369.

†Cf. abstract, Bulletin of the American Mathematical Society, vol. 25 (1922), p. 156. For a statement of Hurwitz's conditions in the case of integral orders of summability see D. S. Morse, Relative inclusiveness of certain definitions of summability, American Journal of Mathematics, vol. 45 (1923), pp. 263, 264. 
only a slight increase of generality in one of these being needed in order to make them both necessary and sufficient. Necessary and sufficient conditions for convergence factors of type $I$ in single series have been given by Kojima.* These conditions, as might be expected, may be obtained from Hurwitz's conditions by dropping the requirement that the convergence factors approach unity as the parameter approaches a certain limit-value. This condition is obviously without significance in the case of convergence factors of type $I$.

Sufficient conditions for convergence factors of type $I$ in double series summable $(C k)$, where $k$ is a positive integer or zero, have been given by the present writer, $\dagger$ and an analogous theorem for triple series summable (C1) has been obtained by Bess M. Eversull. $\ddagger$ The discussion of convergence factors of type II in double series in my paper of 1913 contains an error, and as a result the conditions there given $\S$ are not in themselves sufficient. They can be made so by the addition of conditions $\left(D_{1}\right)$ and $\left(D_{2}\right)$ of Theorem III of this paper.

The purpose of the present paper is to obtain necessary and sufficient conditions for convergence factors of both types in the case of multiple series that are summable $(C k)$, where $k$ is any positive integer or zero. The theorems will be stated both for double series and for multiple series of order $n$. For the sake of simplicity in writing, the proofs will be carried through for the case of double series, the proper changes for the analogous proofs in the case of multiple series of higher order being indicated where this seems desirable. $\|$

For the applications of convergence factor theorems that arise in problems in mathematical physics, most of the various sets of sufficient conditions that have been given are adequate. For such applications, however, as the study of the general theory of series and the relationship between various methods for summing divergent series it seems highly important to have sets of conditions that are both necessary and sufficient.

*Kojima, On generalized Toeplitz's theorems on limit and their applications, Tôhoku Mathematical Journal, vol. 12 (1917), pp. 291-326. See in particular $\$ \S 7,8$. References to previous sets of sufficient conditions for this case may be found in this paper.

†Cf. On convergence factors in double series and the double Fourier's series, these Transactions, vol. 14 (1913), pp. 73-104. See in particular Lemma 4.

‡Cf. Lemma 8 of her paper, On convergence factors in triple series and the triple Fourier's series, Annals of Mathematics, (2), vol. 24 (1922-23). For sufficient conditions for convergence factors of type II in triple series summable (C1) see Theorem II of this paper.

§Loc. cit., Theorem III; and for the special case of series summable (C1), Theorem II.

IIThe reader will of course readily see how to adapt the proof to the case of single series. 
2. Definitions and fundamental identities. The general term in a multiple series of order $n$ may be identified by a set of subscripts $\left(i_{1}, i_{2}, \cdots, i_{n}\right)$. By way of abbreviation, we shall use the symbol [i] to denote this set of subscripts. The series may then be represented by the notation $\sum a_{[i]}$. Using $[m]$ similarly in place of the set of symbols $\left(m_{1}, m_{2}, \cdots, m_{n}\right)$, we set

$$
\begin{aligned}
& S_{[m]}= \sum_{i_{1} n=1}^{m_{1}} \sum_{i_{2}=1}^{m_{2}} \cdots \sum_{i_{n}=1}^{m_{n}} a_{[i]}, \\
& S_{[m]}^{(k)}=\sum_{i_{1}=1}^{m_{1}} \cdots \sum_{i_{n}=1}^{m_{n}} \frac{\Gamma\left(k+m_{1}-i_{1}\right)}{\Gamma(k) \Gamma\left(m_{1}-i_{1}+1\right)} \cdot \frac{\Gamma\left(k+m_{2}-i_{2}\right)}{\Gamma(k) \Gamma\left(m_{2}-i_{2}+1\right)} \\
& \cdots \frac{\Gamma\left(k+m_{n}-i_{n}\right)}{\Gamma(k) \Gamma\left(m_{n}-i_{n}+1\right)} S_{[i]},
\end{aligned}
$$

$$
A_{[m]}^{(k)}=\frac{\Gamma\left(m_{1}+k\right)}{\Gamma(k+1) \Gamma\left(m_{1}\right)} \cdot \frac{\Gamma\left(m_{2}+k\right)}{\Gamma(k+1) \Gamma\left(m_{2}\right)} \cdots \frac{\Gamma\left(m_{n}+k\right)}{\Gamma(k+1) \Gamma\left(m_{n}\right)} .
$$

If the quotient $\left(S_{[m]}^{(\boldsymbol{k})} / A_{[m]}^{(\boldsymbol{k})}\right)$ approaches a limit $S$ as $m_{1}, m_{2}, \cdots, m_{n}$ become infinite, we say that the series is summable $(C k)^{*}$ to the value $S$.

Corresponding to the $r$ th differences of single sequences, we introduce the following definition of the $r$ th difference of a multiple sequence of order $n$ :

(3) $\Delta_{r r \cdots r} f_{[i]}=\sum_{s 1=0}^{r} \cdots \sum_{s n=0}^{r}(-1)^{s_{1}} \cdots(-1)^{s_{n}}\left(\begin{array}{c}r \\ r-s_{1}\end{array}\right) \cdots\left(\begin{array}{c}r \\ r-s_{n}\end{array}\right) f_{[i+\varepsilon]}$,

where it is understood that the subscript $r$ is repeated $n$ times, and that $[i+s]$ stands for the set of numbers $i_{1}+s_{1}, i_{2}+s_{2}, \cdots, i_{n}+s_{n}$. For differences of this type with certain terms missing we use the notationt defined by

$$
\begin{gathered}
\Delta_{\left(r, p_{1}\right)\left(r, p_{2}\right) \cdots\left(r, p_{n}\right)} f_{[i]}=\sum_{s_{1}=0}^{r-p_{1}} \cdots \sum_{s_{n=0}}^{r-p_{n}}(-1)^{s_{1}} \cdots(-1)^{s_{n}}\left(\begin{array}{c}
r \\
r-s_{1}
\end{array}\right) \\
\cdots\left(\begin{array}{c}
r \\
r-s_{n}
\end{array}\right) f_{[i+s]} .
\end{gathered}
$$

*The above definition may be used for any value of $k$ except negative integers. In this paper we shall confine ourselves to the case where $k$ is a positive integer or zero. It is apparent that the definition could be generalized even for this case by introducing $n$ different indices instead of the single index $k$. The appropriate changes to make in the various convergence factor theorems in order to have them apply to this more general case are fairly obvious.

†For the sake of simplicity in writing, a subscript of $\Delta$ of the form $(r, r)$ will be replaced by 0 in subsequent formulas. 
In the case of double series there was obtained in my paper of 1913 a certain fundamental identity, ${ }^{*}$ involving the terms of the series with convergence factors, the expressions (1), and the differences (3) and (4). In our present notation this identity may be written $\dagger$

$$
\begin{gathered}
\sum_{i=1}^{p+r} \sum_{j=1}^{q+r} a_{i j} f_{i j}=\sum_{i=1}^{p} \sum_{j=1}^{q} S_{i j}^{(r-1)} \Delta_{r r} f_{i j}+\sum_{i=1}^{p} \sum_{j=1}^{r} S_{i, q+j}^{(r-1)} \Delta_{r(r, j)} f_{i, q+j} \\
+\sum_{i=1}^{r} \sum_{j=1}^{q} S_{p+i, j}^{(r-1)} \Delta_{(r, i) r} f_{p+i, j}+\sum_{i=1}^{r} \sum_{j=1}^{r} S_{p+i, q+j}^{(r-1)} \Delta_{(r, i)(r, j)} f_{p+i, q+j} .
\end{gathered}
$$

The analogous identity for multiple series of order $n$ is as follows :

$$
\begin{aligned}
& \sum_{i 1=1}^{m_{1}+r} \ldots \sum_{i_{n}=1}^{m_{n}+r} a_{[i]} f_{[i]}=\sum_{i_{1}=1}^{m_{1}} \cdots \sum_{i_{n}=1}^{m_{n}} S_{[i]}^{(r-1)} \Delta_{r} \cdots r f_{[i]} \\
& +\left[\sum_{i_{1}=1}^{m_{1}} \cdots \sum_{i_{n-1}=1}^{m_{n-1}} \sum_{i_{n}=1}^{r} S_{[i, 1, m]}^{(r-1)} \Delta_{r \cdots r\left(r, i_{n}\right)} f_{[i, 1, m]}\right]+\cdots \\
& +\left[\sum_{i=1}^{m_{1}} \cdots \sum_{i_{n-k=1}}^{m_{n-k}} \sum_{i_{n}-k+1=1}^{r} \cdots \sum_{i_{n}=1}^{r} S_{[i, k, m]}^{(r-1)} \Delta_{r} \cdots r\left(r, i_{n-k+1}\right) \cdots\left(r, i_{n}\right) f_{[i, k, m]}\right] \\
& +\cdots+\left[\sum_{i_{1}=1}^{r} \cdots \sum_{i_{n}=1}^{r} S_{[i, n, m]}^{(r-1)} \Delta_{\left(r, i_{1}\right) \cdots\left(r, i_{n}\right)} f_{[i, n, m]}\right] \text {, }
\end{aligned}
$$

where the small bracket symbol $[i, k, m]$, for $k=1,2, \cdots, n$, stands for the set of indices $i_{1}, \cdots, i_{n-k}, m_{n-k+1}+i_{n-k+1}, \cdots, m_{n}+i_{n}$, and the large brackets inclosing an expression indicate the sum of the whole group of terms of similar form, obtained by permutation of indices.

3. Theorems on convergence factors of type $I$. In this section we shall state the theorems of this nature for the case of double series and of multiple series of order $n>2$.

TheOREM I. The necessary and sufficient conditions that the double series $\sum a_{i j} f_{i j}(\alpha, \beta)$ converge for all values of $\alpha$ and $\beta$ included in a set $E(\alpha, \beta)$, whenever the series $\sum a_{i j}$ is summable $(C, r-1)$ and satisfies the condition

$$
\left|S_{i j}^{(r-1)} / A_{i j}^{(r-1)}\right|<C \quad(i, j=1,2, \cdots, C \text { a constant })
$$

are that the convergence factors $f_{i j}(\alpha, \beta)$ satisfy the following conditions:

\footnotetext{
* Loc. cit., pp. $88,89$.

† The use of the index $(r-1)$ instead of $k$ is for the purpose of simplifying the writing of the identity.
} 


$$
\sum_{i=1}^{\infty} \sum_{j=1}^{\infty} i^{r-1} j^{r-1}\left|\Delta_{r r} f_{i j}(\alpha, \beta)\right|<K(\alpha, \beta)
$$

$\left(B_{1}\right) \lim _{j \rightarrow \infty} j^{r-1} \sum_{i=1}^{p} i^{r-1}\left|\Delta_{r 0} f_{i j}(\alpha, \beta)\right|=0$

$$
(E(\alpha, \beta) ; p=1,2, \cdots)
$$

$\left(B_{2}\right) \lim _{i \rightarrow \infty} i^{r-1} \sum_{j=1}^{q} j^{r-1}\left|\Delta_{0 r} f_{i j}(\alpha, \beta)\right|=0$ $(E(\alpha, \beta) ; q=1,2, \cdots) ;$

$$
i^{r-1} j^{r-1}\left|f_{i j}(\alpha, \beta)\right|<M(\alpha, \beta)
$$$$
(E(\alpha, \beta) ; i, j=1,2, \cdots) \text {, }
$$

where $K(\alpha, \beta)$ and $M(\alpha, \beta)$ are finite for each pair of values of $(\alpha, \beta)$ in $E(\alpha, \beta)$.

TheOREM II. The necessary and sufficient conditions that the multiple series $\sum a_{[i]} f_{[i]}\left(\alpha_{1}, \alpha_{2}, \cdots, \alpha_{n}\right)$ converge for all values of $\left(\alpha_{1}, \cdots, \alpha_{n}\right)$ included in a set $E\left(\alpha_{1}, \cdots, \alpha_{n}\right)$, whenever the series $\sum a_{[i]}$ is summable $(C, r-1)$ and satisfies the condition

$$
\left|S_{[i]}^{(r-1)} / A_{[i]}^{(r-1)}\right|<C \quad\left(i_{1}, \cdots, i_{n}=1,2, \cdots ; C \text { a constant }\right),
$$

are that the convergence factors $f_{[i]}\left(\alpha_{1}, \cdots, \alpha_{n}\right)$ satisfy the following conditions:

$$
\begin{aligned}
& \sum_{i_{1}=1}^{\infty} \cdots \sum_{i n=1}^{\infty}\left(i_{1} \cdots i_{n}\right)^{r-1}\left|\Delta_{r \cdots,} f_{[i]}\left(\alpha_{1}, \cdots, \alpha_{n}\right)\right| \\
& <K\left(\alpha_{1}, \cdots, \alpha_{n}\right) \quad\left(E\left(\alpha_{1}, \cdots, \alpha_{n}\right)\right) ;
\end{aligned}
$$

$n$ conditions $\left(B_{8}^{(1)}\right)$ of the type

$$
\begin{aligned}
& \left(B_{1}{ }^{(1)}\right) \quad \lim _{i_{n} \rightarrow \infty} i_{n}^{r-1} \sum_{i_{1}=1}^{p_{1}} \cdots \sum_{i_{n-1}=1}^{p_{n-1}}\left(i_{1} \cdots i_{n-1}\right)^{r-1} \Delta_{r \cdots, r_{0}} f_{[i]}\left(\alpha_{1}, \cdots, \alpha_{n}\right)=0 \\
& \left(E\left(\alpha_{1}, \cdots, \alpha_{n}\right) ; p_{1}, \cdots, p_{n-1}=1,2, \cdots\right) ;
\end{aligned}
$$

$\left(\begin{array}{l}n \\ k\end{array}\right)$ conditions $\left(B_{a^{(k)}}^{(k)}\right)$ of the type

$$
\begin{aligned}
\left(B_{1}(k)\right) \quad & \lim _{\left(i_{n-k+1}, \cdots, i_{n} \rightarrow \infty\right)}\left(i_{n-k+1} \cdots i_{n}\right)^{r-1} \sum_{i_{1}=1}^{p_{1}} \\
& \cdots \sum_{i_{n-k-1}^{p_{n-k}}\left(i_{1} \cdots i_{n-k}\right)^{r-1} \Delta_{r} \cdots, 0 \cdots 0} f_{[i]}\left(\alpha_{1}, \cdots, \alpha_{n}\right)=0 \\
& \left(E\left(\alpha_{1}, \cdots, \alpha_{n}\right) ; p_{1}, \cdots, p_{n-k}=1,2, \cdots\right),
\end{aligned}
$$

where the first $n-k$ subscripts of $\Delta$ have the value $r$, and the $k$ remaining subscripts are equal to $0, k$ ranging from 2 to $n-1$ inclusive;

(C) $\left|\left(i_{1} \cdots i_{n}\right)^{r-1} f_{[i]}\left(\alpha_{1}, \cdots, \alpha_{n}\right)\right|<M\left(\alpha_{1}, \cdots, \alpha_{n}\right)$

$$
\left(E\left(\alpha_{1}, \cdots, \alpha_{n}\right) ; i_{1}, \cdots, i_{n}=1,2, \cdots\right) ;
$$

where $K\left(\alpha_{1}, \cdots, \alpha_{n}\right)$ and $M\left(\alpha_{1}, \cdots, \alpha_{n}\right)$ are finite and positive for each set of values of $\left(\alpha_{1}, \cdots, \alpha_{n}\right)$ in $E\left(\alpha_{1}, \cdots, \alpha_{n}\right)$. 
4. Sufficiency of the conditions. We represent by $S$ the value to which $\sum a_{i j}$ is summable, and we introduce the series $\sum \bar{a}_{i j}$, where $\bar{a}_{11}=a_{11}-S$ and the other $\bar{a}$ 's are identical with the corresponding $a$ 's. If we form $\bar{S}_{i j}^{(r-1)}$ from the series $\sum \bar{a}_{i j}$ in the same way that $S_{i j}^{(r-1)}$ was formed from $\sum a_{i j}$, we have an identity of the form (5) between the $\bar{a}_{i j}$ and the $\bar{S}_{i j}^{(r-1)}$. Since $\bar{S}_{i j}^{(r-1)} /(i j)^{r-1}$ remains finite for all values of $(i, j)$ and approaches zero as $i$ and $j$ become infinite, it follows from conditions $(A),\left(B_{1}\right),\left(B_{2}\right)$, and $(C)$ respectively that the first, second, third, and fourth terms* on the right hand side of (5) approach limits as $p$ and $q$ become infinite. Hence the left hand side of (5) approaches a limit under the same conditions, and the sufficiency of our conditions is established.

If we allow $p$ and $q$ to become infinite in the identity of the form (5), we obtain the following identity: $\dagger$

$$
\sum_{i=1}^{\infty} \sum_{j=1}^{\infty} \bar{a}_{i j} f_{i j}(\alpha, \beta)=\sum_{i=1}^{\infty} \sum_{j=1}^{\infty} \bar{S}_{i j} \Delta_{r r} f_{i j}(\alpha, \beta) \quad(E(\alpha, \beta)) .
$$

5. Necessity of the conditions. We consider first condition $(C)$. If $(C)$ were not satisfied for a certain choice of $\alpha$ and $\beta$, we could find a set of values of $i$ and $j,\left(p_{1}, q_{1}\right),\left(p_{2}, q_{2}\right), \cdots,\left(p_{n}, q_{n}\right), \cdots$, such that $p_{n+1}-p_{n}$ and $q_{n+1}-q_{n}$ are greater than or equal to $(r+1)$ for every $n$, and for which $\left|(i j)^{r-1} f_{i j}(\alpha, \beta)\right|=M_{i j}$ becomes infinite as $i$ and $j$ become infinite by taking on these values successively. We then consider the series for which $S_{i j}^{(r-1)}=(i j)^{r-1} / M_{i j}^{1 / 2}\left(i=p_{1}, p_{2}, \cdots ; j=q_{1}, q_{2}, \cdots\right)$ and $S_{y j}^{(r-1)}=0$ for all other values of $i$ and $j$. For this series

$$
a_{i j}=\Delta_{r r} S_{i-r, j-r}^{(r-1)}=(i j)^{r-1} / M_{i j}^{1 / 2} \quad\left(i=p_{1}, p_{2}, \cdots ; j=q_{1}, q_{2}, \cdots\right) .
$$

Hence $\left|a_{i j} f_{i j}\right|=M_{i j}^{1 / 2}\left(i=p_{1}, p_{2}, \cdots ; j=q_{1}, q_{2}, \cdots\right)$, and therefore $a_{i j} f_{i j}$ does not approach a limit as $i$ and $j$ become infinite. Thus we have a contradiction, since the series $\sum a_{i j} f_{i j}$ was supposed to be convergent.

We pass next to condition $(A)$. If $(A)$ does not hold for a certain choice of $\alpha$ and $\beta,\left(\alpha_{1}, \beta_{1}\right)$, we can select a set of values of $p, p_{1}, p_{2}, \cdots, p_{n}, \cdots$, such that if we define

$$
\sigma_{p}=\sum_{i=1}^{p} \sum_{j=1}^{p}(i j)^{r-1}\left|\Delta_{r r} f_{i j}\left(\alpha_{1}, \beta_{1}\right)\right|
$$

*In the case of multiple series of order $n>2$ the additional terms on the right hand side of the identity (6) are taken care of by means of the additional conditions of the form $B_{a}{ }^{(k)}$.

IIt should be noted that an identity of this form holds for any series $\sum a_{i j}$ summable $(C, r-1)$ to zero, if the factors $f_{i j}$ satisfy conditions $(A),\left(B_{1}\right),\left(B_{2}\right)$, and $(C)$. 
we shall have

$$
\sigma_{p_{n+1}}-\sigma_{p_{n+r}}>1 \quad(n=1,2, \cdots) .
$$

We may then obtain a contradiction from the identity (5) by using the special series for which

$$
\begin{aligned}
& S_{i j}^{(r-1)}=\left[\operatorname{sgn} \Delta_{r r} f_{i j}\left(\alpha_{1}, \beta_{1}\right)\right] \frac{(i j)^{r-1}}{n} \\
&\left(p_{n}+r<{ }_{j}^{i} \leqq p_{n+1} ; 1 \leqq\right. \\
& j
\end{aligned}
$$

$S_{i j}^{(r-1)}=0$ for all other values of $(i, j)$. For this series is summable $(C, r-1)$ to the value zero and satisfies condition ( 7$)$. Therefore $\sum a_{i j} f_{i j}\left(\alpha_{1}, \beta_{1}\right)$ is convergent, or the left hand side of (5) approaches a limit for $\alpha=\alpha_{1}$, $\beta=\beta_{1}$, as $p$ and $q$ become infinite. But it is readily seen that with the above choice of $S_{i j}^{(r-1)}$ the right hand side of (5) becomes infinite when $p$ and $q$ become infinite by taking on the special set of values $\left(p_{2}, q_{2}\right),\left(p_{3}, q_{3}\right), \cdots$, $\left(p_{n}, q_{n}\right), \cdots$. From this contradiction the necessity of $(A)$ follows at once.

Since the proofs for the necessity of $\left(B_{1}\right)$ and $\left(B_{2}\right)$ are entirely analogous, we shall deal only with $\left(B_{1}\right)$. In the case of multiple series of order $n>2$, the proof of the necessity of each of the $\left(2^{n}-2\right)$ conditions $\left(B_{s}^{(k)}\right)$ is analogous to the proof here given for condition $\left(B_{1}\right)$ of Theorem I.

If $\left(B_{1}\right)$ does not hold for a certain choice of $\alpha$ and $\beta,\left(\alpha_{1}, \beta_{1}\right)$, we can find an $\epsilon>0$ and an $m$ such that

$$
q^{r-1} \sum_{i=1}^{m} i^{r-1}\left|\Delta_{r 0} f_{i q}\left(\alpha_{1}, \beta_{1}\right)\right|>\epsilon
$$

for an infinite number of choices of $q, q_{1}, q_{2}, \cdots, q_{n}, \cdots$, each one of which exceeds the preceding one by at least $r$.

We then consider the series for which

$$
\begin{array}{rr}
S_{i q_{n}}^{(r-1)}=(-1)^{n}\left(i q_{n}\right)^{r-1}\left[\operatorname{sgn} \Delta_{r 0} f_{i q_{n}}\left(\alpha_{1}, \beta_{1}\right)\right] & (1 \leqq i \leqq m, n=1,2, \cdots), \\
S_{i j}^{(r-1)}=0 & \text { (all other } i, j) .
\end{array}
$$

This series is summable $(C, r-1)$ to the value zero and satisfies condition ( 7$)$. If we allow $p$ and $q$ to become infinite in such a manner that $q$ takes on the successive values $q_{n}-r(n=1,2, \cdots)$, the second term on the right hand side of (5) will oscillate between values $>\epsilon$ and $<-\epsilon$. The other terms on the right hand side, and the left hand side, will approach definite limits. Thus we shall have a contradiction, and the necessity of $\left(B_{1}\right)$ is established. 
Since, as we have already pointed out, the necessity of $\left(\dot{B}_{2}\right)$ may be proved in analogous fashion, it follows that all four of the conditions $(A)$, $\left(B_{1}\right),\left(B_{2}\right)$, and $(C)$ are necessary in order that every series summable $(C, r-1)$ and satisfying condition (7) may be reduced to convergence by the introduction of the convergence factors $f_{i j}(\alpha, \beta)$.

6. Theorems on convergence factors of type II. We will now state the two theorems of this kind for the case of double series and of multiple series of order $n>2$.

TheOREM III. The necessary and sufficient conditions that the double series $\sum a_{i j} f_{i j}(\alpha, \beta)$ should converge in $E(\alpha, \beta)$ and should approach $S$ as $(\alpha, \beta) \rightarrow\left(\alpha_{0}, \beta_{0}\right)$, a limit point of $E(\alpha, \beta)$ not included in that set, whenever the series $\sum a_{i j}$ is summable $(C, r-1)$ to the value $S$ and satisfies condition (7), are that the convergence factors $f_{i j}(\alpha, \beta)$ satisfy the conditions of Theorem I and the following further conditions:

$$
\sum_{i=1}^{\infty} \sum_{j=1}^{\infty} i^{r-1} j^{r-1}\left|\Delta_{r r} f_{i j}(\alpha, \beta)\right|<K \quad\left(E^{\prime}(\alpha, \beta)\right)
$$

$$
\lim _{(\alpha, \beta) \rightarrow\left(\alpha_{0}, \beta_{0}\right)} \sum_{j=q}^{\infty} j^{r-1}\left|\Delta_{r r} f_{i j}(\alpha, \beta)\right|=0 \quad(i, q=1,2, \cdots),
$$

$$
\begin{array}{ll}
\lim _{(\alpha, \beta) \rightarrow\left(\alpha_{0}, \beta_{0}\right)} \sum_{i=p}^{\infty} i^{r-1}\left|\Delta_{r r} f_{i j}(\alpha, \beta)\right|=0 & (p, j=1,2, \cdots), \\
\lim _{(\alpha, \beta) \rightarrow\left(\alpha_{0}, \beta_{0}\right)} f_{i j}(\alpha, \beta)=1 & (i, j=1,2, \cdots),
\end{array}
$$

where $K$ is a positive constant and $E^{\prime}(\alpha, \beta)$ includes all points of $E(\alpha, \beta)$ lying in a certain neighborhood of $\left(\alpha_{0}, \beta_{0}\right)$.

Theorem IV. The necessary and sufficient conditions that the multiple series $\sum a_{[i]} f_{[i]}\left(\alpha_{1}, \alpha_{2}, \cdots, \alpha_{n}\right)$ should converge in $E\left(\alpha_{1}, \cdots, \alpha_{n}\right)$ and should approach $S$ as $\left(\alpha_{1}, \cdots, \alpha_{n}\right) \rightarrow\left(\alpha_{1}{ }^{(0)}, \cdots, \alpha_{n}{ }^{(0)}\right)$, a limit point of $E\left(\alpha_{1}, \cdots, \alpha_{n}\right)$ not included in that set, whenever the series $\sum a_{[i]}$ is summable $(C, r-1)$ to the value $S$ and satisfies condition (8), are that the convergence factors $f_{[i]}\left(\alpha_{1}, \cdots, \alpha_{n}\right)$ satisfy the conditions of Theorem II and the following further conditions:

$$
\begin{array}{r}
\sum_{i_{1}=1}^{\infty} \cdots \sum_{i_{n}=1}^{\infty}\left(i_{1} \cdots i_{n}\right)^{r-1}\left|\Delta_{r \cdots, r} f_{[i]}\left(\alpha_{1}, \cdots, \alpha_{n}\right)\right|<K \\
\left(E^{\prime}\left(\alpha_{1}, \cdots, \alpha_{n}\right)\right),
\end{array}
$$


$n$ conditions $\left(D_{s}\right)$ of the type

$\left(D_{1}\right) \quad \lim _{\left(\alpha_{1}, \cdots, \alpha_{n}\right) \rightarrow\left(\alpha_{1}^{(0)}, \cdots, \alpha_{n}^{(0)}\right)} \sum_{i_{2}-q_{2}}^{\infty} \cdots \sum_{i_{n}-q_{n}}^{\infty}\left(i_{2} \cdots i_{n}\right)^{r-1}\left|\Delta_{r \cdots r} f_{[i]}\left(\alpha_{1}, \cdots, \alpha_{n}\right)\right|=0$

and

$$
\left(i_{1}, q_{2}, \cdots, q_{n}=1,2, \cdots\right)
$$

$$
\lim _{\left(\alpha_{1}^{\prime}, \cdots, \alpha_{n}\right) \rightarrow\left(\alpha_{1}^{(0)}, \cdots, \alpha_{n}^{(0)}\right)} f_{[i]}\left(\alpha_{1}, \cdots, \alpha_{n}\right)=1 \quad\left(i_{1}, \cdots, i_{n}=1,2, \cdots\right),
$$

where $K$ is a positive constant and $E^{\prime}\left(\alpha_{1}, \cdots, \alpha_{n}\right)$ includes all points of $E\left(\alpha_{1}, \cdots, \alpha_{n}\right)$ lying in a certain neighborhood of $\left(\alpha_{1}{ }^{(0)}, \cdots, \alpha_{n}{ }^{(0)}\right)$.

7. Sufficiency of the conditions. If we introduce the notation $\bar{a}_{i j}$ and $\bar{S}_{i j}$ with the same 'significance as in the proof of Theorem I, the identity (9) follows from conditions $(A),\left(B_{1}\right),\left(B_{2}\right)$, and $(C)$. We will show that under conditions $\left(A^{\prime}\right),\left(D_{1}\right),\left(D_{2}\right)$, and $(E)$ the right hand side of (9) approaches zero as $(\alpha, \beta) \rightarrow\left(\alpha_{0}, \beta_{0}\right)$. It will follow that the left hand side of (9) approaches zero under similar circumstances, and that therefore $\sum a_{i j} f_{i j}(\alpha, \beta)$ approaches $S$ as $(\alpha, \beta) \rightarrow\left(\alpha_{0}, \beta_{0}\right)$. Thus the sufficiency of our conditions will be established. In the case of multiple series of order $n>2$ the proof follows similar lines, conditions $\left(D_{s}\right)(s=1,2, \cdots, n)$, being used in a manner analogous to the use of conditions $\left(D_{1}\right)$ and $\left(D_{2}\right)$.

Given an arbitrary positive $\epsilon$, we choose $(p, q)$ so that

$$
\left[\bar{S}_{i j}^{(r-1)} /(i j)^{r-1}\right]<(\epsilon / 4 K) \quad(i \geqq p, j \geqq q) .
$$

It then follows from $\left(A^{\prime}\right)$ that

$$
\left|\sum_{i=p}^{\infty} \sum_{j=q}^{\infty} S_{i j}^{(r-1)} \Delta_{r r} f_{i j}(\alpha, \beta)\right|<\frac{\epsilon}{4} \quad\left(E^{\prime}(\alpha, \beta)\right) .
$$

In view of $\left(D_{1}\right)$ and $\left(D_{2}\right)$ and condition (7), it follows that there is a certain neighborhood of $\left(\alpha_{0}, \beta_{0}\right)$ such that for the set $E^{\prime \prime}(\alpha, \beta)$ of $E(\alpha, \beta)$, lying in this neighborhood,

$$
\begin{aligned}
& \left|\sum_{i=1}^{p-1} \sum_{j=q}^{\infty} S_{i j}^{(r-1)} \Delta_{r r} f_{i j}(\alpha, \beta)\right|<\frac{\epsilon}{4} \\
& \left|\sum_{i=p}^{\infty} \sum_{j=1}^{q-1} S_{i j}^{(r-1)} \Delta_{r r} f_{i j}(\alpha, \beta)\right|<\frac{\epsilon}{4}
\end{aligned} \quad\left(E^{\prime \prime}(\alpha, \beta)\right) .
$$


From $(E)$ it follows that a set $E^{\prime \prime \prime}(\alpha, \beta)$, lying in $E(\alpha, \beta)$, may be found such that

$$
\left|\sum_{i=1}^{p-1} \sum_{j=1}^{q-1} S_{i j}^{(r-1)} \Delta_{r r} f_{i j}(\alpha, \beta)\right|<\frac{\epsilon}{4} \quad\left(E^{\prime \prime \prime}(\alpha, \beta)\right) .
$$

If we represent by $\bar{E}(\alpha, \beta)$ the set of points that is common to $E^{\prime}, E^{\prime \prime}$, and $E^{\prime \prime \prime}$, we have from (10), (11), and (12),

$$
\left|\sum_{i=1}^{\infty} \sum_{j=1}^{\infty} \bar{S}_{i j}^{(r-1)} \Delta_{r r} f_{i j}(\alpha, \beta)\right|<\epsilon \quad(\bar{E}(\alpha, \beta)) .
$$

Hence $\sum \bar{S}_{i j}^{(r-1)} \Delta_{r r} f_{i j}(\alpha, \beta) \rightarrow 0$ as $(\alpha, \beta) \rightarrow\left(\alpha_{0}, \beta_{0}\right)$ over values included in $E(\alpha, \beta)$, and as pointed out above, the sufficiency of our conditions is established.

8. Necessity of the conditions. We consider first condition $(E)$. Choose a double series $\sum a_{i j}$ which is summable $(C, r-1)$ to a value $S \neq 0$, and represent by $\sum \bar{a}_{i j}$ the series for which $\bar{a}_{m n}=a_{m n}-S, a_{m n}$ being any term of $\sum a_{i j}$, and the other terms $\bar{a}_{i j}$ are identical with the terms $a_{i j}$. It then follows that the series $\sum \bar{a}_{i j}$ is summable $(C, r-1)$ to zero, and hence by our hypothesis the series $\sum a_{i j} f_{i j}(\alpha, \beta)$ and $\sum \bar{a}_{i j} f_{i j}(\alpha, \beta)$ are each convergent in $E(\alpha, \beta)$. We have obviously

$$
\sum \bar{a}_{i j} f_{i j}(\alpha, \beta)+S f_{m n}(\alpha, \beta)=\sum a_{i j} f_{i j}(\alpha, \beta) \quad(E(\alpha, \beta)) .
$$

If we let $(\alpha, \beta) \rightarrow\left(\alpha_{0}, \beta_{0}\right)$, it follows from our hypothesis that the first term on the left hand side of this equation approaches zero and that the right hand side approaches $S$. Hence the second term on the left hand side approaches $S$ as a limit as $(\alpha, \beta) \rightarrow\left(\alpha_{0}, \beta_{0}\right)$, and consequently

$$
\lim _{(\alpha, \beta) \rightarrow\left(\alpha_{0}, \beta_{0}\right)} f_{m n}(\alpha, \beta)=1 \quad(m, n=1,2, \cdots) .
$$

Thus the necessity of $(E)$ is established.

Consider next $\left(A^{\prime}\right)$. For a double series which is summable $(C, r-1)$ to zero, we have the identity

$$
F(\alpha, \beta)=\sum_{i=1}^{\infty} \sum_{j=1}^{\infty} a_{i j} f_{i j}(\alpha, \beta)=\sum_{i=1}^{\infty} \sum_{j=1}^{\infty} S_{i j}^{(r-1)} \Delta_{r r} f_{i j}(\alpha, \beta),
$$

whenever the series in the second member converges in $E(\alpha, \beta)$ and condition (7) is satisfied. For by Theorem I conditions $(A),\left(B_{1}\right),\left(B_{2}\right)$, and $(C)$ are necessarily satisfied under the hypotheses stated, and therefore the above identity holds.* If $\left(A^{\prime}\right)$ is not satisfied for a certain set $E^{\prime}(\alpha, \beta)$,

*Cf. the second footnote, $\$ 4$. 
having $\left(\alpha_{0}, \beta_{0}\right)$ as a limit point, we can select from $E(\alpha, \beta)$ a sequence of $(\alpha, \beta)$ 's, $\left(\alpha^{(m)}, \beta^{(m)}\right)$, approaching $\left(\alpha_{0}, \beta_{0}\right)$ and such that

$$
\sum_{i=1}^{p} \sum_{j=1}^{p}(i j)^{r-1}\left|\Delta_{r r} f_{i j}\left(\alpha^{(m)}, \beta^{(m)}\right)\right|>m \quad(m=1,2, \cdots)
$$

for a proper choice of $p$. Choose $m_{1} \geqq 3$, and let $\left(\alpha_{1}, \beta_{1}\right)$ and $p_{1}$ be the corresponding values of $(\alpha, \beta)$ and $p$. Take $s_{1}>p_{1}$, and define

$$
S_{i j}^{(r-1)}=\left[\operatorname{sgn} \Delta_{r r} f_{i j}\left(\alpha_{1}, \beta_{1}\right)\right](i j)^{r-1} \quad\left(1 \leqq_{j}^{i} \leqq s_{1}\right) .
$$

Now choose $\left(\alpha_{2}, \beta_{2}\right)$ from the sequence $\left(\alpha^{(m)}, \beta^{(m)}\right)$ such that*

$$
\sum_{i=1}^{s_{1}} \sum_{j=1}^{s_{1}}(i j)^{r-1}\left|\Delta_{r r} f_{i j}\left(\alpha_{2}, \beta_{2}\right)\right|<\frac{1}{4 \log m_{1}}
$$

and let $m_{2}$ and $p_{2}$ be the corresponding values of $m$ and $p$ for which the inequality (13) holds. Take $s_{2}>p_{2}$ and such that

$$
\sum_{i=1}^{\infty} \sum_{j=1}^{\infty}(i j)^{r-1}\left|\Delta_{r r} f_{i j}\left(\alpha_{2}, \beta_{2}\right)\right|-\sum_{i=1}^{s_{2}} \sum_{j=1}^{s_{2}}(i j)^{r-1}\left|\Delta_{r r} f_{i j}\left(\alpha_{2}, \beta_{2}\right)\right|<\frac{1}{4 \log m_{1}} .
$$

We then define

$$
S_{i j}^{(r-1)}=\left[\operatorname{sgn} \Delta_{r r} f_{i j}\left(\alpha_{2}, \beta_{2}\right)\right] \frac{(i j)^{r-1}}{\log m_{1}} \quad\left(\begin{array}{c}
s_{1}<i \leqq s_{2}, 1 \leqq j \leqq s_{2} \\
1 \leqq i \leqq s_{1}, s_{1}<j \leqq s_{2}
\end{array}\right) .
$$

Continuing in this fashion, we define a series which is summable $(C, r-1)$ to zero, while at the same time $\left|F\left(\alpha_{n}, \beta_{n}\right)\right|>\left(m_{n} / 4 \log m_{n-1}\right)(n=2,3, \cdots)$, so that $F(\alpha, \beta)$ does not tend to a limit as $(\alpha, \beta) \rightarrow\left(\alpha_{0}, \beta_{0}\right)$ over any set in $E(\alpha, \beta)$. Thus we have a contradiction and the necessity of $\left(A^{\prime}\right)$ is established.

The proofs of the necessity of $\left(D_{1}\right)$ and $\left(D_{2}\right)$ are entirely analogous, and we will therefore consider only the case of $\left(D_{1}\right)$. In the case of multiple series of order $n>2$ the proof for each of the various conditions $\left(D_{\varepsilon}\right)$ is analogous to the proof for $\left(D_{1}\right)$ here given.

If $\left(D_{1}\right)$ does not hold, we can find an $\epsilon$, an $m$, and a $q$ such that

$$
\sum_{j=q}^{\infty} j^{r-1}\left|\Delta_{r r} f_{m j}(\alpha, \beta)\right|
$$

*That such an $\left(\alpha_{2}, \beta_{2}\right)$ exists follows from the necessity of $(E)$, already established. 
exceeds $\epsilon$ for an infinite set of values of $(\alpha, \beta)$ lying in $E(\alpha, \beta)$ and having $\left(\alpha_{0}, \beta_{0}\right)$ as a limit point. Choose one pair of values $\left(\alpha_{1}, \beta_{1}\right)$ from this sequence and determine $s_{1}$ such that

$$
\sum_{j=q}^{s_{1}} j^{r-1}\left|\Delta_{r r} f_{m j}\left(\alpha_{1}, \beta_{1}\right)\right|>\frac{3 \epsilon}{4}, \sum_{j=s_{1}+1}^{\infty} j^{r-1}\left|\Delta_{r r} f_{m j}\left(\alpha_{1}, \beta_{1}\right)\right|<\frac{\epsilon}{4} .
$$

Then take

$$
S_{m i}^{(-1)}=\left[\operatorname{sgn} \Delta_{r r} f_{m j}\left(\alpha_{1}, \beta_{1}\right)\right] j^{r-1} \quad\left(q \leqq j \leqq s_{1}\right) .
$$

Now find $\left(\alpha_{2}, \beta_{2}\right)$ such that

$$
\sum_{j=q}^{s_{1}} j^{r-1}\left|\Delta_{r r} f_{m j}\left(\alpha_{2}, \beta_{2}\right)\right|<\frac{\epsilon}{8}
$$

and choose $s_{2}$ so that

$$
\sum_{j=\alpha_{1+1}}^{\delta_{2}} j^{r-1}\left|\Delta_{r r} f_{m j}\left(\alpha_{2}, \beta_{2}\right)\right|>\frac{3 \epsilon}{4}, \sum_{j=\Omega 2+1}^{\infty} j^{r-1}\left|\Delta_{r r} f_{m j}\left(\alpha_{2}, \beta_{2}\right)\right|<\frac{\epsilon}{8} .
$$

Then take

$$
S_{m i}^{(r-1)}=\left[\operatorname{sgn} \Delta_{r r} f_{m j}\left(\alpha_{2}, \beta_{2}\right)\right] j^{r-1} \quad\left(s_{1}<j \leqq s_{2}\right) .
$$

Continue this process for the choice of $S_{m j}^{(r-1)}(j \geqq q)$, and choose all other $S_{i j}^{(r-1)}=0$. Thus we shall obtain a series which is summable $(C, r-1)$ to zero, whereas $F\left(\alpha_{n}, \beta_{n}\right)>\frac{1}{2} \epsilon(n=1,2, \cdots)$. This contradiction of our hypothesis establishes the necessity of $\left(D_{1}\right)$, and as stated above the necessity of $\left(D_{2}\right)$ can be proved in a manner entirely analogous.

UnIVERSity of Cincinnatr,

Cincinnati, OHio 